\title{
Surface Transport in Pre-Melted Films with Application to Grain-Boundary Grooving
}

\author{
Robert W. Style and M. Grae Worster \\ Institute of Theoretical Geophysics, Department of Applied Mathematics and Theoretical Physics, \\ University of Cambridge CB3 $9 E W$ Cambridge, England
}

\begin{abstract}
We present a new model of surface transport in premelted films that is applicable to a wide range of materials close to their melting point. We illustrate its use by applying it to the evolution of a grain boundary groove in a high vapour pressure material and show that Mullins's classical equation describing transport driven by gradients in surface curvature is reproduced asymptotically. The microscopic contact angle at the groove root is found to be modified over a thin boundary layer, and the apparent contact angle is determined. An explicit transport coefficient is derived that governs the evolution rate of systems controlled by surface transport through premelted films. The transport coefficient is found to depend on temperature and diverges as the bulk melting temperature is approached.
\end{abstract}

It is well established [? ] that melting in any material is initiated at the free surface. Thus, a molecularly thin layer of melt liquid can exist at the surface at temperatures below the bulk transition temperature, depending on the predominant intermolecular interactions at the solid-vapour interface. Typically, repulsive van der Waals forces or electrostatic interactions act to thicken the film, while the film width is constrained by an attractive pressure arising from the liquid being held below its freezing point. (For a wide ranging review, see Dash et al. [1].) Wettlaufer and Worster 2] have shown that it is possible to use lubrication theory to model the flow in the film, and they found that their results were in good agreement with experiment, using bulk parameter values (e.g. dynamic viscosity) in the flow [3]. Furthermore, there exists much evidence, both theoretical [4], [5] and experimental [6] showing that the continuum fluid dynamical approach, combined with bulk parameters can be used for films thicker than about ten molecular diameters. Therefore, in systems with sufficiently thick films, we can derive a system of continuum equations to model the evolution of solid-vapour surfaces near to their melting point.

In this letter, we derive the equations pertaining to surface melting and flow in a surface-melted film. We then apply these to the evolution of a grain-boundary (GB) groove and demonstrate that, in the long time limit, the original equation derived by Mullins [7]

$$
y_{t}+B y_{x x x x}=0,
$$

is recovered asymptotically. Here, $y(x, t)$ is the surface height, $x$ is distance parallel to the surface and $B$ is a constant transport parameter. We use typical bulk parameters for the water/ice system at $-1^{\circ}$ to give predictions of grooving rates that are in agreement with typical observations. We focus on ice because it is experimentally accessible and because there is much research on it due to its unique importance in geoscience. It is also known to premelt against air [8], and may be doped to yield sufficiently thick films to justify the continuum approach 9].

Firstly, using the assumption that the system is in local thermodynamic equilibrium, we have as a consequence of the Gibbs-Duhem relationship [10] that

$$
p_{s}-p_{l}=\rho_{s} q_{m}\left(\frac{T_{m}-T}{T_{m}}\right),
$$

where $\rho, p, T, T_{m}$ and $q_{m}$ are density, pressure, system temperature, melting temperature and latent heat of fusion respectively, and subscripts $s$ and $l$ correspond to the solid and liquid phases. We have also used the assumption that the system is isothermal.

Defining $h_{1}(x, t)$ to be the height of the solid-liquid surface, $h_{2}(x, t)$ to be the height of the liquid-air surface, $d(x, t)=h_{2}-h_{1}$ to be the thickness of the liquid layer (cf Fig. 1 for a specific example), $\gamma_{s l}$ and $\gamma_{l a}$ to be the (constant) surface free energies of the two interfaces, and $A$ to be the effective Hamaker constant 2], we have that the Helmholtz free energy

$$
\begin{gathered}
F=\int_{0}^{\infty}\left[-p_{s}\left(h_{2}-d\right)-p_{l} d+p_{a} h_{2}+\gamma_{l a}\left(1+h_{2}^{\prime 2}\right)^{1 / 2}\right. \\
\left.+\left(\gamma_{s l}+\frac{A}{12 \pi d^{2}}\right)\left(1+h_{1}^{\prime 2}\right)^{1 / 2}\right] d x
\end{gathered}
$$

Treating the integrand as a function of $h_{1}$ and $d$ and minimising $F$ using the Euler-Lagrange equation gives

$$
p_{s}-p_{l}=\frac{A}{6 \pi d^{3}}\left(1+h_{1}^{\prime 2}\right)^{1 / 2}+\left(\gamma_{s l}+\frac{A}{12 \pi d^{2}}\right) K_{1}
$$

where $K_{1}$ is the curvature of the solid-liquid interface $z=h_{1}$.

We assume that the thickness of the film is much greater than a characteristic molecular dimension, and that the slopes of the surfaces are small. Therefore

$$
\gamma_{s l} \gg \frac{A}{12 \pi d^{2}}, \quad h_{1}^{\prime \prime} \ll 1,
$$


and we can approximate

$$
p_{s}-p_{l}=\frac{A}{6 \pi d^{3}}-\gamma_{s l} h_{1}^{\prime \prime}
$$

which we combine with Eq. (2) to give a general result for a surface-melted film (cf [1] )

$$
\frac{A}{6 \pi d_{0}^{3}} \equiv \rho_{s} q_{m}\left(\frac{T_{m}-T_{0}}{T_{m}}\right)=-\gamma_{s l} h_{1}^{\prime \prime}+\frac{A}{6 \pi d^{3}} .
$$

The thickness of the layer is determined by the competition between the rise in energy required to sustain a liquid film below the melting point, and the van der Waals $(\mathrm{VdW})$ forces, as expressed in the balance between the LHS and last term on the RHS in (7). As can also be seen in the equation, the Gibbs-Thomson effect manifests itself in that an increase in surface curvature causes an increase in the thickness of the film.

The second Euler-Lagrange relationship from above yields

$$
p_{l}=p_{a}-\gamma_{l a} h_{2}^{\prime \prime}-\frac{A}{6 \pi d^{3}}
$$

So, in regions where the film is thick and has high curvature, the liquid pressure is high. This drives flow away, towards regions where the curvature is lower and the film thinner.

Finally, assuming that the thickness of the film is sufficiently small, we can use lubrication theory [11] to give the velocity in the layer (see Fig. (1) insert) using a noslip condition at the solid-liquid interface and a stressfree condition at the liquid-air interface. Mass conservation is expressed by

$$
\frac{\partial q}{\partial x}+\dot{h}_{2}=0
$$

where $q(x)=-d^{3} p_{x} /(3 \mu)$, and $\mu$ is the dynamic viscosity of water.

We introduce dimensionless variables with a lengthscale determined by taking the balance between $\mathrm{VdW}$ and curvature terms in equation (7) to give

$$
\begin{aligned}
& x=\left(\frac{A}{6 \pi \gamma_{s l}}\right)^{1 / 2} \xi \equiv \epsilon \xi \\
& t=\left(\frac{3 \mu^{2} A}{2 \pi \gamma_{s l}^{3}}\right)^{1 / 2} \tau=\delta \tau
\end{aligned}
$$

and we write $\left(h_{1}, h_{2}, d, d_{0}\right)=\epsilon\left(\mathcal{H}_{1}, \mathcal{H}, \mathcal{D}, \mathcal{D}_{0}\right)$.

Thus we obtain a coupled pair of dimensionless partial differential equations

$$
\frac{1}{\mathcal{D}_{0}^{3}}-\frac{1}{\mathcal{D}^{3}}=-\mathcal{H}^{\prime \prime}+\mathcal{D}^{\prime \prime}
$$

$$
\left[\mathcal{D}^{3}\left(\gamma \mathcal{H}^{\prime \prime \prime}+\left(\frac{1}{\mathcal{D}^{3}}\right)^{\prime}\right)\right]^{\prime}+\dot{\mathcal{H}}=0
$$

where $\gamma=\gamma_{l a} / \gamma_{s l}$. Boundary conditions for these equations depend on the specific problem to be addressed.

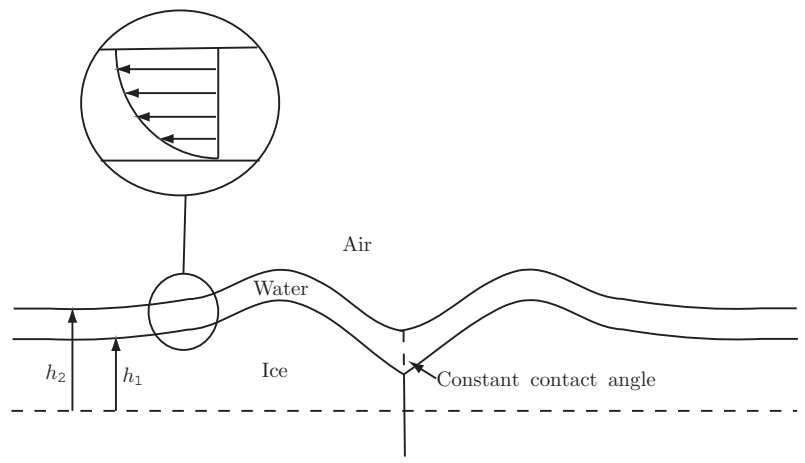

FIG. 1: Schematic diagram of a grain-boundary groove. The system is isothermal with $T=T_{0}$.

We can now, for example, address the problem of a grain-boundary groove. The model to be analysed is shown in Fig. 1]. Two ice crystals, symmetric about the GB between them are exposed to the air. The system is maintained at a uniform temperature $T_{0}$ sufficiently close to the melting point $T_{m}$ that surface melting occurs and a liquid film is formed, separating solid from air. Initially, the upper surfaces of both crystals are planar, but equilibrium demands a fixed dihedral angle (given by the Young-Dupré relationship [12]), at the tri-junction formed by the GB and the solid-liquid interfaces. The premelted film is thereby made thicker near the GB, the disjoining force across it is correspondingly weaker and the liquid pressure higher. This drives a flow in the premelted film transporting mass outwards to form a GB groove.

Using this model, we take boundary conditions to equations (12), (13) to be $\mathcal{D}(\infty)=\mathcal{D}_{0}, \mathcal{H}^{\prime}(0)=0$, $\mathcal{D}^{\prime}(0)=-\alpha$ and $q(0)=0$, corresponding respectively to constant film thickness at infinity, constant contact angle at the groove base (with cotangent $\alpha$ ), continuous derivative of the liquid-air interface above the groove and zero mass flux across the plane of symmetry.

As time proceeds, the groove widens and deepens, while the thickness of the premelted film stays relatively constant. Therefore we can make the assumption (justifiable a posteriori) that $\mathcal{D} \ll \mathcal{H}$ and hence $\mathcal{D}^{\prime \prime} \ll \mathcal{H}^{\prime \prime}$ away from a neighbourhood of the $z$ axis. Thus (12) becomes

$$
\frac{1}{\mathcal{D}_{0}^{3}}-\frac{1}{\mathcal{D}^{3}}=-\mathcal{H}^{\prime \prime}
$$

Therefore, assuming that $\mathcal{D} \approx \mathcal{D}_{0}$ (see below) and com- 
bining with (13), we recover the Mullins equation

$$
\mathcal{H}_{\tau}+\mathcal{D}_{0}^{3}(\gamma+1) \mathcal{H}_{\xi \xi \xi \xi}=0,
$$

with dimensional transport coefficient

$$
B \equiv \frac{A(\gamma+1) \gamma_{s l}}{18 \pi \mu \rho_{s} q_{m}} \frac{T_{m}}{\left(T_{m}-T_{0}\right)} .
$$

This equation admits a similarity solution [7]

$$
\mathcal{H}_{s}=\left[\mathcal{D}_{0}^{3}(\gamma+1) \tau\right]^{1 / 4} f(\eta)
$$

where

$$
\eta=\frac{\xi}{\left[\mathcal{D}_{0}^{3}(\gamma+1) \tau\right]^{1 / 4}} .
$$

and the function $f$ satisfies

$$
f^{\mathrm{iv}}+\frac{f}{4}-\frac{\eta f^{\prime}}{4}=0
$$

subject to the boundary conditions $f^{\prime \prime \prime}(0)=0, f^{\prime}(0)=\beta$, $f^{\prime}(\infty)=0$ and $f(\infty)=0$. This yields $\mathcal{H}_{s}(0)$ which we will use to match to the inner boundary layer solution. The constant $\beta$ is the cotangent of the apparent contact angle seen by this outer region, and will be determined by matching to the inner asymptotic solution, derived as follows.

In the similarity solution regime, we take $\mathcal{H} \sim \tau^{1 / 4} f(\eta)$ and $\mathcal{D} \sim g(\eta)$. Combining these estimates with Eq. (12) yields $\mathcal{D}=\mathcal{D}_{0}+O\left(\tau^{1 / 4}\right)$ and hence the similarity solution is expected to break down at the GB when $\mathcal{D}^{\prime} \rightarrow-\alpha$.

In this regime, we assume that the $\tau^{1 / 4}$ time dependence of the similarity solution is carried over into the inner solution. Then consideration of the order of magnitude of terms in Eq. (13) shows that $\mathcal{H}_{\tau}$ vanishes for large times, and we can make quasi-steady approximations of $\mathcal{H}(\xi, \tau)=C \tau^{1 / 4}+H(\xi)$ and $\mathcal{D}(\xi, \tau)=D(\xi)$ inside the boundary layer.

Applying a zero flux boundary condition at the GB, Eq. (13) becomes

$$
D^{3}\left[\gamma H^{\prime \prime \prime}+\left(\frac{1}{D^{3}}\right)^{\prime}\right]=0,
$$

which we integrate with $H^{\prime \prime}(\infty)=D^{\prime \prime}(\infty)=0, D^{\prime}(0)=$ $-\alpha$ and $W^{\prime}(\infty)=0$ to obtain

$$
\mathcal{H}_{\text {inner }}=C \tau^{1 / 4}+\frac{D}{1+\gamma}+\frac{\alpha \xi}{1+\gamma}+c,
$$

where, from Eq. (12), $D$ satisfies the equation

$$
(1+\gamma) D^{\prime \prime}=\gamma\left(\frac{1}{\mathcal{D}_{0}^{3}}-\frac{1}{D^{3}}\right)
$$

Substituting $\mathcal{H}_{s}=\tau^{1 / 4} f(\eta)$ into Eq. (13), we find that as $\tau \rightarrow \infty, \mathcal{H}_{s}$ satisfies the full equation. Therefore we
TABLE I: Table of typical values for ice/water/air at $T=-1^{\circ}$

\begin{tabular}{|l|r|r|}
\hline Constant & Value & Units \\
\hline$\rho_{s}$ & 917 & $\mathrm{~kg} \mathrm{~m}^{-3}$ \\
$q_{m}$ & $3.34 \times 10^{5}$ & $\mathrm{~J} \mathrm{~kg}^{-1}$ \\
$\gamma_{s l}$ & $3 \times 10^{-2}$ & $\mathrm{~J} \mathrm{~m}^{-2}$ \\
$\gamma_{l a}$ & $7.57 \times 10^{-2}$ & $\mathrm{~J} \mathrm{~m}^{-2}$ \\
$A$ & $1.50 \times 10^{-20}$ & $\mathrm{~J}$ \\
$\epsilon$ & $5.16 \times 10^{-10}$ & $\mathrm{~m}$ \\
$\delta$ & $2.9 \times 10^{-11}$ & $\mathrm{~s}$ \\
$d_{0}$ & $8.93 \times 10^{-10}$ & $\mathrm{~m}$ \\
$w_{0}$ & 1.73 & - \\
$\mu$ & $1.787 \times 10^{-3}$ & $\mathrm{~kg} \mathrm{~m}^{-1} \mathrm{~s}^{-1}$ \\
$B$ & $1.40 \times 10^{-26}$ & $\mathrm{~m}^{4} \mathrm{~s}^{-1}$ \\
\hline
\end{tabular}

can match Eqs. (17) and (21) by letting $\mathcal{H}_{\text {inner }}(\xi) \rightarrow \mathcal{H}_{s}$ and $D(\xi) \rightarrow \mathcal{D}_{0}$ for large $\xi$ (the outer edge of the inner solution), and matching this to the small $\xi$ limit of Eq. (15).

For small $\xi$, Eq. (17) becomes

$$
\mathcal{H}_{s}=\mathcal{H}_{s}(0)+\xi \beta+O\left(\tau^{-1 / 4}\right)
$$

giving that

$$
\mathcal{H}_{\text {inner }}=\mathcal{H}_{s}(0)+\frac{D-\mathcal{D}_{0}}{1+\gamma}+\frac{\alpha \xi}{1+\gamma} .
$$

Importantly, we have a value for the apparent cotangent

$$
\beta=\frac{\alpha}{1+\gamma} \text {. }
$$

Eq. (25) shows that for $\gamma_{l a}$ large relative to $\gamma_{s l}$, the system will try to minimize the liquid-air interfacial area, and hence $\beta \rightarrow 0$. Conversely for $\gamma_{s l} \gg \gamma_{l a}, \beta \rightarrow \alpha$ as the solid-liquid interfacial curvature is minimized at the GB to reduce surface area. This also implies that the apparent cotangent $\beta$ can differ substantially from the actual cotangent $\alpha$ suggesting that experimental determination of $\alpha$ could yield incorrect results due to the apparent contact angle being measured instead.

To solve the complete system of equations, we used an explicit numerical scheme. At each step, the $\mathcal{D}$ profile is calculated from (12) by a relaxation technique. The new value of $\mathcal{H}$ at $(\tau+d \tau)$ can then be calculated from (13), using the previous values of $\mathcal{H}(\tau)$ and $\mathcal{D}(\tau)$.

Fig. 2 shows the time evolution of the groove. Good agreement can be seen, even at small times, between the numerical solutions (continuous lines) and the similarity solution (dashed lines). Boundary conditions for the similarity solution are $\mathcal{H}^{\prime}(0)=\beta, q(0)=0, \mathcal{H}(\infty)=0$ and $\mathcal{H}^{\prime}(\infty)=0$. The inset figure shows the inner boundary layer region for $t=2.9 \times 10^{-4}$. The inner asymptotics (dash-dot) are shown, and also show good agreement with the numerical solution. 


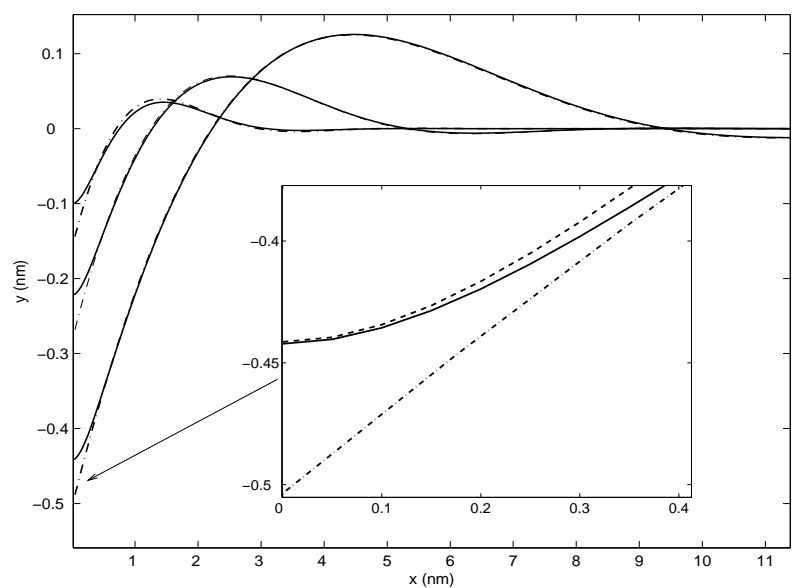

FIG. 2: Numerical solutions (continuous lines) and similarity solutions (dashed lines) for time evolution of a groove at a liquid-air interface. $y$ is the the height of the interfaces relative to the initial position. Graphs are plotted in terms of $x$ and $t$ at $t=8.7 \times 10^{-7}, 1.7 \times 10^{-5}, 2.9 \times 10^{-4}$. Inset shows numerical solution (continuous line), similarity solution (dashed line) and inner boundary layer asymptotics (dash-dot line) for the inner boundary layer regime at $t=2.9 \times 10^{-4}$. Parameters were chosen as $\mathcal{D}_{0}=1, \gamma=0.5$.

From Eq.(16) we see that the rate-controlling coefficient is inversely proportional to the reduced temperature, and grows as $T_{0}$ approaches the melting point. We also see that less viscous, less dense liquids with thicker layers ( $A$ large) and stronger surface tension cause faster grooving than more viscous, dense liquids with thin layers and weak surface tension. Typical parameters for ice at $-1^{\circ}$ (see Table I) give $B$ to be approximately $1.4 \times 10^{-26} \mathrm{~m}^{4} \mathrm{~s}^{-1}$ and so typical lengthscales (proportional to $\left.\left(B t^{1 / 4}\right)\right)$ are of order $0.35 \mu \mathrm{m}$ in $1 \mathrm{~s}$ and $3.5 \mu \mathrm{m}$ in 3 hours, which is in line with observations of ice [13]. At this time, the authors are unaware of any experimental measurements of $B$ in any material within a suitable temperature range.

The analysis presented above is relevant to any material that premelts against its atmosphere and forms a constant contact angle at the groove root, in circumstances where it is maintained at a suitable temperature that premelting can occur. However, several points should be noted. (i) We have assumed that the slope of the interfaces is always small, and therefore some detail of the form of the groove root may have been lost. (ii) We assumed that there is a complete phase change across the surface $z=\mathcal{H}_{1}$, while the transition may actually occur across several atomic diameters and involve some level of ordering(eg [? ]). However, provided the premelted film is thick enough, the affects of this should be negligible and we may use continuum approximations. Appropriately thick films may be ensured by the addition of dopants to the system [9]. (iii) We have considered surface melting controlled by unretarded VdW forces. This can be easily generalised to retarded VdW forces and electrostatic interactions [3]. (iv) We require $\alpha$ to be small for the lubrication approximation to be valid in the inner region. However, groove root angles for ice may be very small, so this analysis can break down near the groove root.

So, in conclusion, we have demonstrated a new mechanism for mass transport on the surface of a crystal in a suitably high temperature regime. Surface melting is a ubiquitous phenomena, and so the equations that we have derived should be applicable to a wide range of materials and situations. We have illustrated the mechanism of surface transport by applying it to the case of a grain-boundary groove and demonstrated that it yields Mullins's equation in the asymptotic limit, although the dihedral angle determined by the surface energies is modified over the boundary layer by a factor $1 /(1+\gamma)$. We note that much richer behaviour will result from effects such as crystal anisotropy, impurity content, asymmetry and other grooving mechanisms [7].

The most important result of this letter is that we have found an explicit transport parameter $B$ (Eq. (16)) that controls the rate of surface transport in the system. It should be noted that $B$ does not depend on the geometry of the grain-boundary groove. Therefore this parameter should be relevant to most problems involving surface melting such as sintering, grain-boundary groove migration, crystal growth and annealing to name but a few.

The authors would like to thank J.S.Wettlaufer for his critical readings of the letter.

[1] J. G. Dash, H. Fu, and J. S. Wettlaufer, Rep. Prog. Phys. 58, 115 (1995).

[2] J. S. Wettlaufer and M. G. Worster, Phys. Rev. E 51, 4679 (1995).

[3] J. S. Wettlaufer, M. G. Worster, L. A. Wilen, and J. G. Dash, Phys. Rev. Lett 76, 3602 (1996).

[4] K. P. Travis and K. E. Gubbins, J. Phys. Chem. 112, 1984 (2000).

[5] J. Koplik and J. R. Banavar, Annual Review of Fluid Mechanics 27, 257 (1995).

[6] U. Raviv and J. Klein, Science 297, 1540 (2002).

[7] W. W. Mullins, J. Appl. Phys. 28, 333 (1957).

[8] M. Elbaum, S. G. Lipson, and J. G. Dash, J. Cryst. Growth 129, 491 (1993).

[9] L. Benatov and J. S. Wettlaufer, Phys. Rev. E 70, 061606 (2004).

[10] S. E. Wood and R. Battino, Thermodynamics of Chemical Systems (CUP, Cambridge, 1990).

[11] G. K. Batchelor, An Introduction to Fluid Dynamics (Cambridge University Press, 1967).

[12] L. D. Landau and E. M. Lifshitz, Statistical Physics (Pergamon Press, London, 1958).

[13] P. R. F. Barnes, Ph.D. thesis, Open University (2002).

[14] J. S. Wettlaufer, Phil. Trans. R. Soc. Lond. A 357, 3403 (1999). 